\title{
Locally Advanced Large-Cell Neuroendocrine Carcinoma of the Uterine Cervix: A Case Report
}

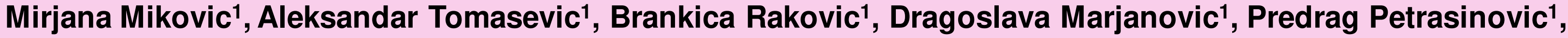 Vesna Plesinac Karapandzic ${ }^{1,2}$}

1Department of Radiotherapy, Clinic for Radiation Oncology and Diagnostics, Institute for Oncology and Radiology of Serbia, Belgrade, Serbia

${ }^{2}$ Faculty of Medicine, University of Belgrade, Belgrade, Serbia

\section{$>$ Introduction}

- Large-cell neuroendocrine carcinoma (LCNEC) of the uterine cervix is rare (up to $0,3 \%$ of cases) and a very aggressive type of tumor even at an early stage. Despite a multimodal treatment approach, a locally advanced stage is often associated with extremely poor prognosis ${ }^{1,2}$.

\section{$>$ Case report}

- Patient age 45 at diagnosis, nullipara, was presented with a large $8 \mathrm{~cm}$, stage IIlb (FIGO) tumor mass of the cervix.

Multiple biopsies patohystologically and immunohistochemically verified LCNEC tumor built of solid epithelioid cells with prominent mitotic activity (Figure 1.). IHH staining was positive for p16, CK AE1/AE3, PGP 9.5 (diffuse expression, Figure 2.), CK7, synaptophysin (focal expression strong intensity), Ki 67 60\%; negative for vimentin, p63, CK5/6, CD 56, Chromogranin A and NSE markers.

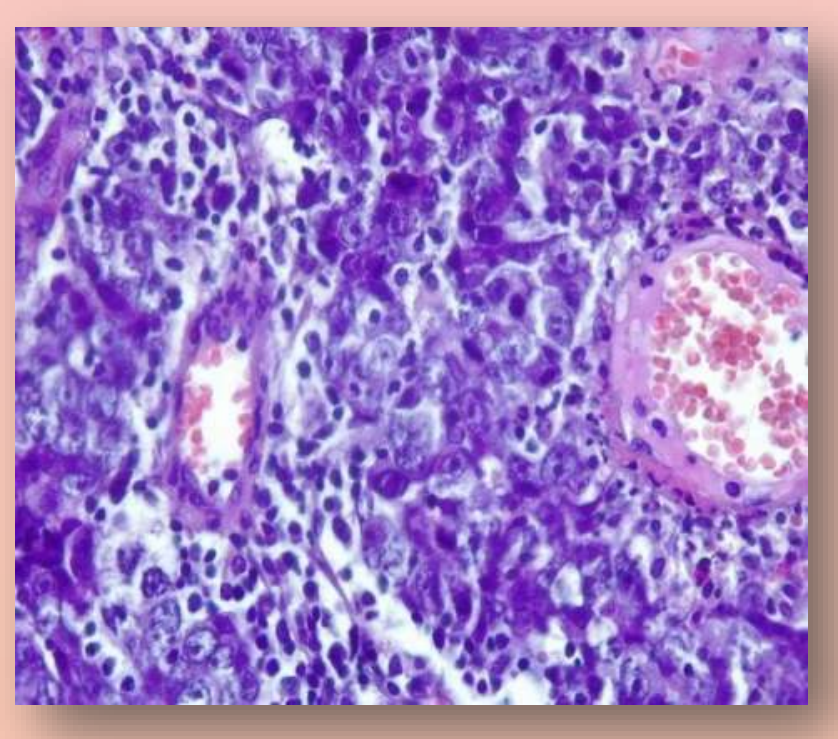

Figure 1. Histology: polygonal cells with eosinophilic cytoplasm, round vesicular nucleuses with prominent nucleoli, chromatin and numerous mitoses. Orig. magnification $\times 40$

- Initial diagnostics: MRI of the abdomen and pelvis revealed the large pelvic mass, dimensions $9 \times 8 \mathrm{~cm}$, (LLXAP, Figure 3. $a, b)$ and two liver hemangiomas. The brain CT was normal. Chest CT showed several micronodular lesions (with calcifications), which were, by diagnostic parameters, considered as insignificant.

- Treatment: definitive radiotherapy with total transcutaneous (TRT) dose of 46 Gy was delivered to the whole pelvis in 25 fractions, concomitant (CCRT) with 5 cycles of weekly cisplatin-based chemotherapy $\left(40 \mathrm{mg} / \mathrm{m}^{2}\right)$ and combined with 5 intracavitary brachytherapy applications (central tube and two ovoids, weekly) with a dose of 7 Gy to reference point A/per application, ending in December 2017.
C CRT effect was estimated as gross partial regression (GPR) with clinically visible residual $\mathrm{Tu}(1,5 \mathrm{~cm})$ at the end of therapy

- Treatment continued: systemic Cisplatin-Etoposide-based chemotherapy regiment (CDDP $75 \mathrm{mg} / \mathrm{m}^{2}$, Etoposide $100 \mathrm{mg} / \mathrm{m}^{2} / 3$ days), 6 cycles, every 3 weeks, ending June 2018.

Follow-up: first post-therapy diagnostic evaluation - chest CT scan showed no progression, MRI of abdomen and pelvis (Figure 4. a, b) showed no signs of initial disease.
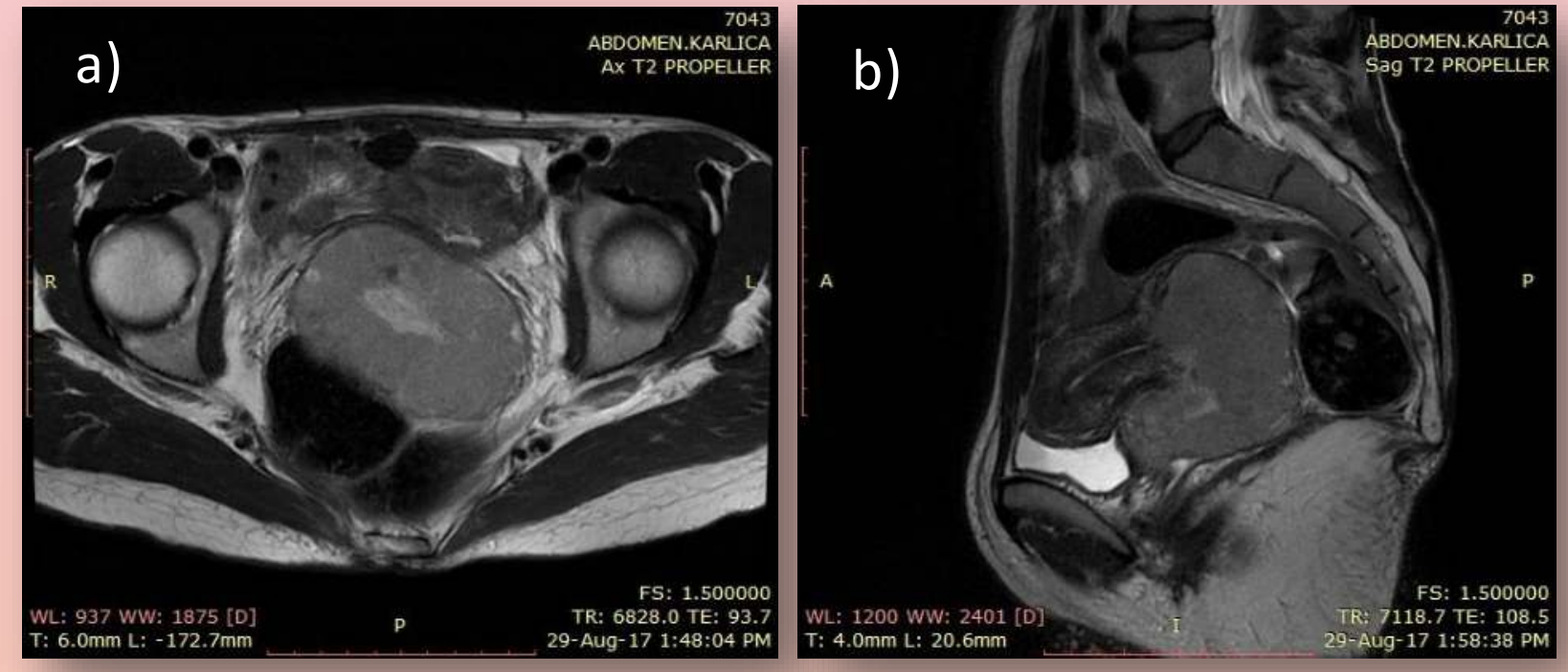

Figure 3. Pretherapy MRI of the pelvis: T2W axial (a) and T2W sagittal (b)
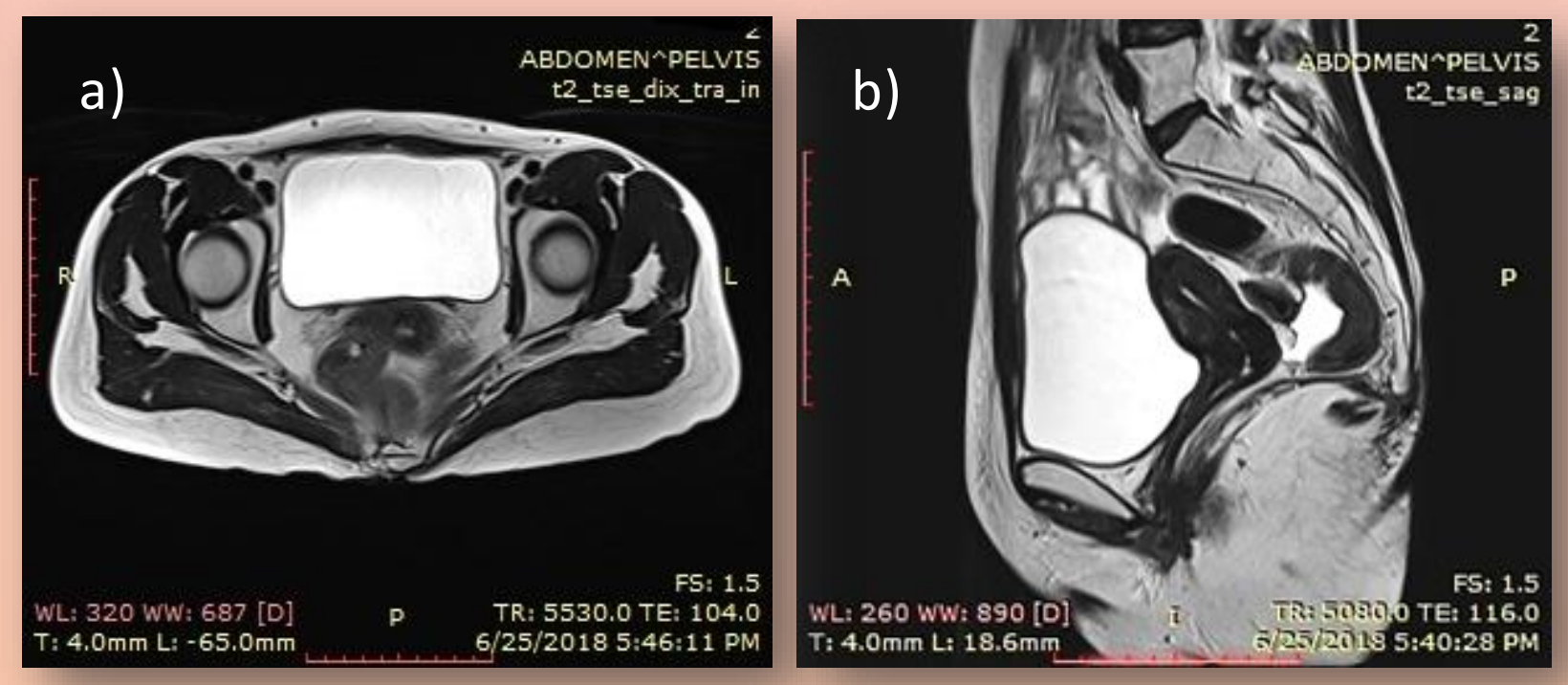

Figure 4. Post-therapy MRI of the pelvis: T2W axial (a) and T2W sagittal (b)

Now, 13 months after diagnosis, the patient is in excellent condition and with no clinical signs of the disease. Excision biopsy was performed on $5 \mathrm{~mm}$ clinically suspicious malignant cervical lesion - microscopic necrotic fragments with inflammatory cells were found, without malignant signs.

> Conclusion: Definitive concomitant radio-chemotherapy seems to be an effective treatment modality in a locally advanced LCNEC tumor; however, further systemic chemotherapy and close monitoring are necessary due to an aggressive form of the disease.

\section{References:}

1. Yoseph B, Chi M, Truskinovsky AM, Dudek AZ. Large-cell neuroendocrine carcinoma of the cervix. Rare Tumors. 2012;4(1), 58-60.

2. Boruta II DM, Schorge JO, Duska LA, Crum CP, Castrillon DH, Sheets EE. Multimodality therapy in early-stage neuroendocrine carcinoma of the 